Service social

\title{
Approche intégrée : une innovation dans la dispensation des services sociaux
}

\section{René Auclair et Christiane Lampron}

Volume 36, numéro 2-3, 1987

Approches intégrées

URI : https://id.erudit.org/iderudit/706365ar

DOI : https://doi.org/10.7202/706365ar

Aller au sommaire du numéro

Éditeur(s)

École de service social de l'Université Laval

ISSN

1708-1734 (numérique)

Découvrir la revue

Citer cet article

Auclair, R. \& Lampron, C. (1987). Approche intégrée : une innovation dans la dispensation des services sociaux. Service social, 36(2-3), 315-341.

https://doi.org/10.7202/706365ar
Résumé de l'article

Cet article synthétise une recherche sur l'approche intégrée effectuée dans le contexte du Centre de services sociaux de Québec, succursale de Charlevoix. Les objectifs de cette recherche visaient à acquérir des connaissances plus précises sur les concepts théoriques d'un mode d'approche intégrée et à approfondir avec les intervenants leur pratique professionnelle.

Après avoir esquissé les contextes de pratique, de théorie et de méthodologie, les auteurs font ressortir les principaux éléments de l'approche intégrée telle que pratiquée à cet endroit, ainsi que les avantages et les difficultés qu'elle soulève. 
AuClaIR, René, professeur à l'École de service social de l'Université Laval.

LAMPRON, Christiane, étudiante au doctorat, Sciences de l'éducation, Université Laval.

\section{Approche intégrée : une innovation dans la dispensation des services sociaux}

\section{René Auclair Christiane Lampron}

Le présent article est une synthèse de la recherche sur l'approche intégrée ${ }^{1}$ effectuée entre juin 1985 et septembre 1987 dans les municipalités de Charlevoix, desservies par le Centre de services sociaux de Québec. Nous y présentons les objectifs du projet de même que la démarche des praticiens à l'origine de cette recherche. Une section consacrée à la méthodologie vient en préciser les cadres théorique et dynamique. On relate brièvement la définition de la problématique à l'étude et les principaux résultats se dégageant de la cueillette de données. Le lecteur ${ }^{2}$ peut enfin prendre connaissance des approches intégrées selon les principales dimensions étudiées en consultant le tableau-synthèse présenté à la fin de l'article (voir Appendice A).

\section{Objectifs de la recherche}

Les objectifs inhérents à la recherche sont essentiellement de trois ordres :

- acquérir des connaissances plus précises sur les concepts théoriques d'un mode d'approche intégré, pour en arriver à une compréhension plus claire de ces notions fondamentales et à une pratique consciente et efficace.

- approfondir avec les intervenants leur pratique professionnelle en tentant d'identifier et d'analyser avec eux les modes d'agir qui structurent leur façon d'intervenir. 
— identifier de façon précise les zones souhaitables de progrès et approfondir l'intervention selon les besoins principaux identifiés.

\section{Démarche de concertation d'équipe}

Différents événements relevés dans le vécu professionnel des intervenants semblent avoir provoqué un renouveau de la pratique du service social dans Charlevoix.

En effet, le discours des intervenants révèle que la situation qui prévalait au C.S.S.Q.-Charlevoix ${ }^{3}$ avant l'année 1976 ressemblait sensiblement à celle souvent décrite et critiquée dans la littérature récente. Outre l'étendue du territoire à couvrir qui générait de nombreuses pertes de temps, les intervenants ont parlé principalement de leurs difficultés d'intervenir à l'intérieur d'une pratique axée presque exclusivement sur le cas à cas, les rendant ainsi captifs d'interventions ponctuelles et partielles. Obligés de répondre à une multitude de besoins provenant de problématiques diversifiées et souvent complexes, ils éprouvaient très souvent le sentiment de manquer de temps pour assurer à leurs clientèles un service professionnel adéquat et de qualité. De plus, le manque de perfectionnement, d'encadrement sur le plan professionnel, de partage avec les autres praticiens ainsi que la grande mobilité du personnel favorisaient le travail en solitaire et le désir de tout régler par soi-même, sans le support et l'aide de ses pairs, d'où essoufflement et épuisement professionnel. Peu à peu, le travail perdait de son intérêt et s'inscrivait à l'intérieur d'une routine dénuée de toute créativité, caractérisée par un éparpillement des énergies. Les intervenants se sentaient de plus en plus dépassés par les événements, isolés professionnellement et géographiquement, voire même désabusés et aliénés. "S'ils ont développé une sorte de corporatisme peu enclin aux changements structurels, ils étaient tout de même à la recherche de personne(s)( ressource(s) qui les ferait (feraient) sortir de leur léthargie professionnelle" . 4 L'arrivée d'un nouveau chef d'équipe au secteur familleenfance leur permit de faire l'expérience de l'approche intégrée. Celuici, principal initiateur de cette démarche, a précisé qu'il faut distinguer trois étapes au cours desquelles s'est effectuée la mise en application de l'approche intégrée : 1) la réappropriation théorique ; 2) la réorganisation de la charge de travail ; 3) la réappropriation pratique ou l'approfondissement de la qualité de l'intervention. 


\section{Mise en application de l'approche intégrée}

La réappropriation théorique s'est appuyée sur une analyse de la situation vécue par les intervenants. Cette démarche a permis de dégager une grille d'analyse de la réalité sociale qui tient compte de l'influence des facteurs structurels sur les problèmes vécus par les usagers des services sociaux de leur région. Cette vision implique également la reconnaissance de l'autonomie des personnes, perçues comme étant en mesure d'organiser leur vie en collaboration avec d'autres. Une telle orientation nécessite, en outre, un choix fondamental pour les "sans voix".

La deuxième étape, ou la réorganisation de la charge de travail, a consisté à briser l'isolement vécu par les intervenants, en travaillant à deux sur certains projets, dans le but de se fournir mutuellement support et critique. De plus, un regroupement des dossiers a permis d'effectuer une nouvelle division du travail en fonction des champs d'intérêt des intervenants.

Finalement, la réappropriation pratique a permis d'approfondir la qualité de l'intervention à partir des expériences vécues. C'est dans cette perspective que furent organisées des sessions de perfectionnement.

C'est donc un mouvement de concertation d'équipe, incluant les intervenants et le chef d'équipe du C.S.S.Q.-Charlevoix, qui est à l'origine de la présente recherche. Des constats communs quant aux limites des interventions à caractère individuel prodiguées aux populations cibles, ont contribué à déclencher la réflexion de ce groupe. Puis, animés d'une volonté partagée de mieux connaître et desservir les besoins de la population, les membres de l'équipe ont convenu d'unifier temps et expertise pour la réalisation de ce projet.

\section{Définition de la problématique}

Comment définir l'approche intégrée ? Voilà un concept fort abstrait qu'on ne saurait expliquer en quelques mots. Afin de présenter le plus clairement possible les paramètres à l'étude et d'éviter les écarts d'interprétation théorique, une classification des modes d'approche intégrés susceptibles d'avoir une incidence sur la solution des problèmes sociaux a été dressée en début de recherche. Cette classification, résultant d'une synthèse des publications, a permis de répertorier quatre principaux modes d'approche intégrés en service social. Ces modes d'approche sont les suivants : le modèle centré sur la tâche, le modèle systémique, 
la perspective psychosociale et le modèle structurel. Cette classification, bien que non exhaustive, a servi de point de référence tout au long de la recherche. Schématiquement, la philosophie d'intervention intégrée peut être présentée de la façon suivante :
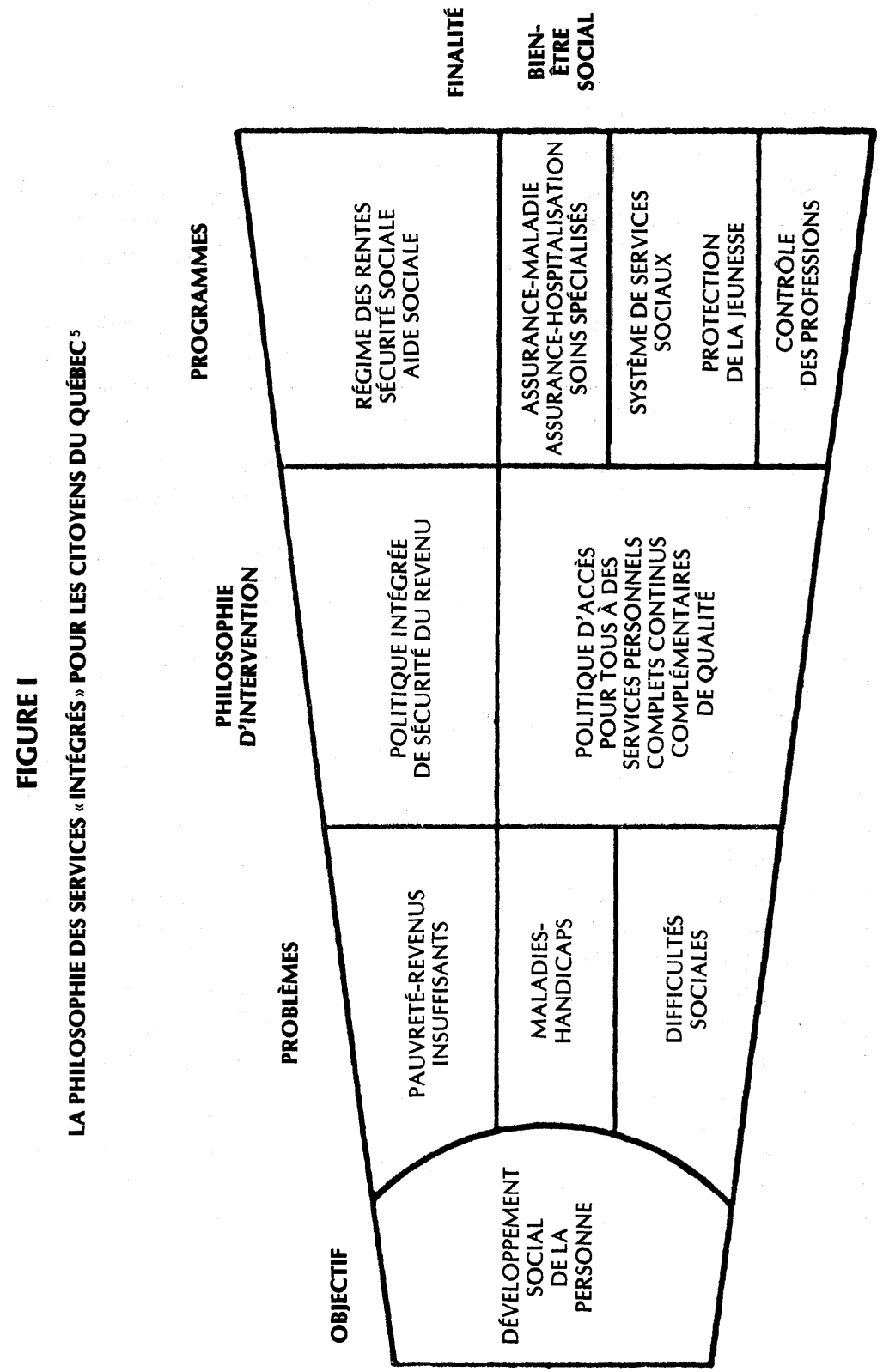


\section{Méthodologie}

Dans la poursuite des objectifs précédemment cités, les méthodes retenues comme cadre de travail sont la recherche documentaire et l'entrevue.

Afin de cerner la documentation sur le mode d'approche intégré en service social, l'équipe de recherche a privilégié une étude documentaire visant à l'exhaustivité.

Le repérage des publications s'est effectué rétrospectivement depuis l'année 1965, à partir de plusieurs bassins documentaires : service social et sciences sociales en général. Cet inventaire a permis de développer :

- une bibliographie commentée des documents répertoriés (publication séparée);

- une revue des publications. Cette analyse détaillée vise à dégager les orientations émanant d'un mode d'approche intégré en service social (revue qui fait l'objet d'un autre article dans le présent numéro).

Cette double démarche, en plus d'alimenter les étapes ultérieures de développement du projet, combe un vide documentaire en service social.

Un guide d'entrevue, couvrant les éléments principaux d'un mode d'approche intégré, comme les objectifs poursuivis, les modes d'intervention ou les résultats obtenus, fut préparé et prétesté. Ce guide a servi de base aux entrevues avec les praticiens de Charlevoix (voir Appendice B). D'autre part, il semblait important de rencontrer, en plus des praticiens, des gestionnaires de divers niveaux hiérarchiques afin d'obtenir d'eux des renseignements qui puissent corroborer ou infirmer les informations venues des praticiens ou, du moins, des opinions qui puissent être mises en parallèle avec celles des praticiens.

Le guide d'entrevue destiné aux gestionnaires cherche à explorer les questions suivantes: objectifs de l'approche intégrée, rôle joué par le gestionnaire, opinions sur l'approche intégrée, possibilités de généralisation (voir Appendice $\mathrm{C}$ ).

Les entrevues avec les praticiens et les gestionnaires ont eu lieu de janvier à avril 1986. Une chargée de recherche a assumé seule la conduite des entrevues auprès des praticiens et, en coresponsabilité avec le directeur de cette étude, la conduite des entrevues auprès des gestionnaires.

La méthode d'analyse que nous avons retenue s'inspire du bilan d'intervention habituellement utilisé dans les organisations. Cette opération consiste à évaluer l'écart entre une situation de départ non 
satisfaisante et une situation d'arrivée satisfaisante au plan organisationnel. La comparaison entre les deux situations peut porter soit sur les résultats, soit sur les processus. ${ }^{6}$

Ne pouvant brosser un tableau complet de l'état antérieur de la situation, nous avons décidé de faire un bilan qui porte plutôt sur les aspects méthodologiques de cette intervention que sur les résultats. Pour ce faire, nous avons comparé certaines dimensions qui caractérisaient l'approche intégrée développée dans Charlevoix (but, rôle, focus et composantes de l'action) à celles des quatre approches présentées au début, soit les approches " centrée sur la tâche, systémique, psychosociale et structurelle ", par l'analyse approfondie des différents critères correspondants. Nous avons choisi d'établir la comparaison avec ces quatre approches parce que d'une part, ce sont celles qui semblaient le plus fréquemment utilisées pendant la période couverte par cette étude dans les différents organismes de prestation de services sociaux et, d'autre part, parce qu'elles avaient fait l'objet d'une analyse critique fort intéressante par différents auteurs américains.

\section{Principaux éléments de l’approche intégrée au C.S.S.Q.-Charlevoix}

\section{Le démarrage de l'expérience}

Soucieux de modifier certaines pratiques qu'ils jugeaient susceptibles de nuire à la qualité des services, les intervenants de Charlevoix se sont penchés sur une façon différente de dispenser les services d'ordre psychosocial dans leur région.

C'est ainsi que, avec le consentement du groupe de praticiens des secteurs famille-enfance et ressources, le premier geste significatif qui a permis d'initier la démarche fut de démarrer l'expérience, sans avoir informé au préalable la direction générale du Centre de services sociaux de Québec. La peur de ne pas recevoir l'autorisation de modifier les pratiques d'intervention qui prévalaient à ce moment-là, la crainte de devoir adapter leur nouveau mode de fonctionnement pour le rendre conforme à l'exercice de certains contrôles administratifs, et le fait aussi que les praticiens relevaient de différentes directions de Québec qui exigeaient un rendement individuel et ne reconnaissaient pas nécessairement l'intervention de groupe et communautaire comme modes d'approche efficaces et pertinents en réponse aux besoins individuels des gens, semblent avoir motivé leur décision. On assistait 
alors à une première prise de position des intervenants face aux structures administratives en place.

Ne pouvant toutefois ignorer l'administration locale de Charlevoix dans la réorganisation de leur service, les intervenants ont cherché à créer une alliance avec cette dernière. On parla alors de cogestion. Pour eux, cela signifiait augmentation du pouvoir, développement d'une force de négociation, partage des responsabilités et de l'information entre les praticiens et la direction. Pour l'administration locale, c'était faire confiance aux professionnels, favoriser le bien-être du personnel et voir à la qualité des services. Concrètement, cela se traduisait par une gestion facilitant la réorganisation du travail des intervenants, tant au niveau des méthodes d'intervention que des conditions matérielles de réalisation.

Ainsi, par l'introduction d'une formule de cogestion, on cherchait à modifier le rôle de l'organisme. Il devint un lieu où des personnes exerçant différentes fonctions travaillent en collaboration pour modifier le modèle organisationnel du travail; favoriser différentes modalités d'intervention; permettre une situation flexible et créatrice des ressources humaines ; encourager une délégation de l'autorité et des responsabilités aux membres du personnel et finalement, faciliter la communication.

\section{Redéfinition du but à atteindre, de la situation-problème et du rôle professionnel}

De cette première décision allaient découler et s'enchaîner une série de modifications tant au niveau de la pratique professionnelle du service social qu'au niveau des attitudes des intervenants. C'est ainsi qu'après avoir pris conscience de leur isolement professionnel, des préjugés qu'ils entretenaient face à différentes clientèles ou problématiques, de leur manque de motivation, de concertation, bref de leur insatisfaction générale à l'égard d'un travail qu'ils avaient à accomplir quotidiennement, ils décidaient d'édifier leur pratique professionnelle sur des bases nouvelles. On commence alors à parler de conscientisation.

Mais pour y arriver, il fallait tout d'abord développer un esprit d'équipe, faire l'apprentissage de la solidarité, prendre conscience de son propre pouvoir dans une situation de prestation de services, d'où la nécessité de modifier certaines pratiques professionnelles et, par conséquent, certaines attitudes et comportements individuels. Graduellement prit place un discours élargi sur la pratique du service social, s'attardant non plus seulement aux seuls échanges de conseils sur la résolution de 
certains cas, mais portant sur les différents aspects de la pratique en général, sur les conditions socio-économiques de la région, sur les causes probables qui pouvaient engendrer les problèmes sociaux auxquels ils étaient confrontés, etc. On sentit alors se renforcer, voire même se développer certaines prises de positions par rapport à la clientèle défavorisée tant financièrement, socialement que psychologiquement et petit à petit émergea une nouvelle vision de la réalité.

L'idéologie qui sous-tend l'expérience de Charlevoix était, aux dires des intervenants, basée sur la théorie de la conscientisation et le but qu'ils poursuivaient découlait directement de cette théorie. Ils cherchaient, par le biais de leurs interventions, à développer un pouvoir collectif et ils luttaient pour la conscientisation. Cette formulation, quoique limitative dans son interprétation à cause d'un manque de précision (ex.: la cible de l'intervention), indique tout de même une volonté ferme d'aller plus loin qu'une simple définition des tâches du client et du professionnel et d'un réaménagement des connaissances et des habiletés requises, tel que le préconise l'approche centrée sur la tâche. C'était plus encore qu'intervenir sur et entre les différents systèmes de façon à créer un impact sur les autres systèmes (approche systémique) ou encore d'intervenir sur les rôles, les structures des petits groupes et sur les processus d'influence sociale (approche psychosociale). Le but réellement visé était d'en arriver à développer des rapports plus égalitaires, tel que le veut l'approche structurelle, par le biais d'un changement radical à moyen et long termes axé sur la lutte des classes et le développement d'un pouvoir collectif. En ce sens, le but poursuivi par les intervenants de Charlevoix, de par son idéologie et les théories explicatives de l'approche structurelle (théorie de l'intégration, du conflit et de la lutte des classes), se rapproche de celui de l'approche structurelle.

Poursuivre un but de conscientisation impliquait donc une analyse de la situation-problème qui mettait l'accent sur les déterminants sociaux des situations. Ainsi, cette analyse s'inspirait d'une vision de l'inadaptation sociale où il était essentiel de tenir compte d'abord et avant tout des conditions socio-économiques et du pouvoir des gens avnt de penser à une intervention à caractère psychologique. Par le fait même, la façon d'interpréter le problème était basée sur une analyse globale des problèmes sociaux où les problèmes individuels étaient perçus comme la manifestation des problèmes sociaux. Cette façon d'interpréter le problème leur permettait alors d'avoir une vue d'ensemble d'un cas pour ensuite choisir les modalités d'interventions les plus susceptibles de résoudre adéquatement la situation. Selon les intervenants, 
une telle analyse orientait le client vers une plus grande responsabilisation et favorisait une meilleure prise en charge collective.

Poursuivre un but de conscientisation impliquait aussi une démarche devant permettre aux usagers des services sociaux de faire ressortir les différents aspects socio-économiques, politiques ou psychologiques de leur situation et favoriser le développement d'actions collectives devant corriger la situation. Cela supposait que les intervenants puissent jouer un rôle de courtier, de médiateur, d'avocat et même d'expert quand ils agissaient comme personne-ressource (approche structurelle). Toutefois, sur ce dernier point, ils se sont entendus pour dire qu'ils agissaient moins à titre d'expert, soit celui qui utilise diverses informations pour produire des changements sur ou avec le client tel que présenté dans l'approche psychosociale, mais davantage comme accompagnateur dans une démarche de conscientisation.

Ainsi, accompagner un individu, un groupe ou une communauté dans une démarche de conscientisation suppose que les intervenants ont été les alliés de la cible d'intervention avec laquelle ils ont pu établir un rapport égalitaire de type dialogique. Cela suppose aussi qu'ils ont été plus que des "facilitateurs" qui aident l'individu, le groupe ou la communauté à réaliser certaines tâches (approche centrée sur la tâche). À cause du contexte dans lequel se déroulait l'action, de l'idéologie qui servait de fondement pour l'action à réaliser et du but qu'ils poursuivaient, le rôle professionnel identifié par les intervenants lors des entrevues présente certaines caractéristiques qui se rapprochent de celles de l'approche structurelle.

\section{Définition de l’approche intégrée}

Dans son sens le plus large, les intervenants définissaient l'approche intégrée comme étant le fait d'utiliser les trois méthodes en service social soit l'individuel, le groupe et le communautaire. Toutefois, ils devaient préciser que l'action envisagée devait évoluer selon un continuum ou parallèlement à différents niveaux, selon la nature des difficultés.

Ainsi, il semblait possible par exemple d'intervenir auprès d'un client qui exprimait des besoins individuels particuliers, de l'amener à cheminer à l'intérieur d'un groupe, pour enfin l'accompagner dans une démarche de conscientisation. À tout moment du processus, il était possible aussi de passer d'une méthode à une autre lorsqu'il s'agissait de répondre à de nouveaux besoins. 


\section{Focus de l'intervention}

Sur cette question, les intervenants ont privilégié trois éléments distincts. Pour eux, il était d'abord important de responsabiliser le client dans sa démarche de résolution de problèmes, le cient étant perçu comme le principal agent de changement et le principal responsable de la résolution de son problème. On considérait aussi que le client possédait les capacités nécessaires pour apporter les changements requis. Il y avait donc un partage du pouvoir avec la clientèle dont le potentiel avait été reconnu. On retrouve ces mêmes valeurs dans l'approche centrée sur la tâche. On parle d'autodétermination du client, de sa croissance et de sa capacité de s'en sortir. Toutes ces valeurs sont déterminantes lorsque l'on met l'accent sur la responsabilisation du client dans la résolution de ses problèmes.

Les intervenants parlaient aussi de créer une alliance avec le client. Ayant reconnu ses potentialités et déterminé les responsabilités qui peuvent être partagées, les intervenants pouvaient plus facilement devenir les alliés de leurs clients. Quoique allant dans le même sens que l'approche structurelle, cet élément semble plus limitatif que ce que prône l'approche elle-même. Elle encourage non seulement la création d'une alliance entre le travailleur social et son client, mais aussi une alliance entre le client et divers groupes sociaux qui travaillent sur une même cause.

Enfin, le fait d'agir au niveau des facteurs structurels et d'avoir pour objectif l'obtention d'un effet multiplicateur vient toutefois élargir l'interprétation de l'élément précédent. Cette action permet alors de désindividualiser les problèmes tout en permettant au client de faire ressortir les aspects socio-politico-économiques de sa situation.

\section{Composantes de l'action professionnelle}

Dès que furent posés les premiers jalons de la réappropriation théorique, les intervenants abordèrent une seconde étape : la réorganisation de la charge de cas. Encore une fois, plusieurs modifications furent apportées et ils purent ainsi remettre en question le mode traditionnel de prestation de services. En travaillant par équipe de deux, trois, voire même davantage sur les mêmes dossiers, on venait de briser l'isolement professionnel dans lequel ils étaient plongés depuis quelques années déjà. Toutefois, la démarche allait prendre tout son sens à travers une nouvelle procédure de répartition des dossiers, basée cette fois sur les intérêts et les aptitudes des intervenants. En effet, il s'agissait non seulement de travailler en équipe sur les mêmes dossiers, mais aussi 
de constituer des équipes dans lesquelles les intervenants partageaient les mêmes intérêts par rapport à une clientèle ou problématique et dont les membres pouvaient présenter des aptitudes ou compétences différentes, le plus souvent complémentaires. Ainsi, ce nouveau mode de travail allait permettre d'enrichir chacune des équipes par des compétences développées individuellement. II allait permettre aussi aux membres de puiser dans l'équipe une énergie nouvelle, un dynamisme renouvelé et l'obligation de se remettre en question, facilitant alors la transition entre l'intervention individuelle et l'approche intégrée, parce que répondant à un besoin de se surpasser et d'améliorer sa pratique.

Seule l'approche centrée sur la tâche parle d'une équipe professionnelle multidisciplinaire formée de représentants des usagers, de travailleurs sociaux, de chercheurs, d'administrateurs et du personnel clérical. Cette équipe a la responsabilité de l'élaboration, de la gestion et de l'évaluation des programmes destinés à la population. Toutefois, l'équipe ne travaille pas ensemble au niveau de l'intervention. On assiste alors au partage de l'autorité entre l'administration, les intervenants et les bénéficiaires.

Quoique s'appuyant sur le travail en équipe, des différences marquées s'observent entre les deux approches (centrée sur la tâche et intégrée) tant au niveau du nombre et de la représentativité de ses membres que du rôle joué par l'équipe. En fait, la différence la plus évidente réside dans le fait que l'équipe multidisciplinaire (approche centrée sur la tâche) soit absente de l'action, tandis que l'on retrouve dans l'approche intégrée une équipe composée de professionnels seulement, œuvrant tout aussi bien au niveau de l'élaboration, de la gestion, de l'action et de l'évaluation de l'action. La première intervient sur les programmes tandis que la seconde se définit dans l'action.

Cette façon de procéder avait exigé au préalable un regroupement des clientèles et une délimitation territoriale. Ainsi, certains intervenants s'intéressaient aux handicapés mentaux, d'autres aux enfants placés avec ou sans ordonnance de la Cour ou encore aux familles présentant des problèmes de fonctionnement, aux chômeurs, etc. Après avoir défini leurs intérêts, pris conscience de leurs capacités, ils pouvaient donc choisir l'une ou l'autre des clientèles et délimiter le territoire sur lequel ils allaient intervenir. L'opération de jumelage se réalisait alors par elle-même.

La notion de territoire était fort importante, puisque chacun des territoires présentait des caractéristiques propres, aussi bien au niveau de la mentalité de sa population, de son éloignement par rapport aux 
centres urbains qu'au niveau de la présence ou de l'absence de ressources adéquates dans le milieu.

Intervenir sur un territoire délimité exigeait donc de la part del'intervenant une bonne connaissance du milieu. Toutefois, cela permettait aussi une intervention basée sur une connaissance plus juste des problématiques habituellement rencontrées sur le territoire, tout en permettant une plus grande visibilité de l'intervenant dans le milieu. Cette présence accrue favorisait son intégration au sein de la communauté, augmentait la confiance des usagers à son égard et stimulait leur participation dans la réalisation du plan d'intervention. Cette nouvelle division du travail allait susciter un vif intérêt parmi les intervenants et fit augmenter la motivation au travail.

Après avoir constitué les équipes, les membres devaient s'appliquer à développer un ou plusieurs projets d'intervention se rapportant à la clientèle retenue. Ce fut l'un des gestes les plus significatifs posé par ces derniers, parce qu'il venait modifier complètement le mode d'intervention de la majorité d'entre eux, soit l'intervention individuelle. C'est ainsi qu'ils furent appelés à travailler autant au niveau des groupes et de la communauté qu'à celui des individus et des familles.

Le fait d'intervenir à différents niveaux fit apparaître de nouvelles tâches : rencontres avec les médias, préparation de réunions, d'assemblées, achat de matériel, etc., occasionnant ainsi une surcharge de travail, mais de meilleurs résultats à plus court terme. Selon les intervenants, les projets ont favorisé l'introduction du volet information de formation de la population, ce qui n'était pas représenté sous cet angle dans un mode traditionnel de prestation de services sociaux.

C'est donc au niveau des différentes composantes de l'action professionnelle que se retrouvent les différences les plus marquées entre l'approche intégrée et les quatre autres approches. En effet, les intervenants mettaient beaucoup d'emphase sur la communauté. On assistait à un important travail de détection, de conscientisation et de formation d'alliés dans la communauté, tout comme on a tenté de mettre sur pied plusieurs ressources (habituellement, l'élément majeur des projets) qui devaient être gérées par la communauté.

Ce travail se réalisait par diade ou triade. Les équipes ainsi formées étaient composées exclusivement d'intervenants sociaux ayant la responsabilité de l'élaboration, de la gestion, de l'action et de l'évaluation de l'action. De plus, chacune des équipes était responsable de l'élaboration d'un ou plusieurs projets d'intervention se rapportant à la clientèle avec laquelle elle avait choisi de travailler. Le projet d'intervention favorisait généralement l'action communautaire et de groupe. Toutefois, 
les bénéficiaires pouvaient aussi recevoir si nécessaire une aide individuelle.

Ainsi, les notions d'équipe, de regroupement de clientèles, de délimitation territoriale et de projet allaient devenir les éléments majeurs de l'action professionnelle des travailleurs sociaux de Charlevoix.

\section{Bref résumé de l'approche intégrée dans Charlevoix}

\section{Définition}

- L'utilisation des trois méthodes en service social, soit l'intervention individuelle, de groupe et communautaire selon un continuum ou parallèlement selon la nature des difficultés.

\section{Analyse de la situation-problème}

- Approche globale;

- Qui permet une vue d'ensemble d'un cas ;

- Qui doit tenir compte d'abord et avant tout des conditions socioéconomiques et du pouvoir des gens;

- Dont les problèmes individuels sont vus comme étant la manifestation des problèmes sociaux.

\section{But du service social}

- Développer un pouvoir collectif ;

- Lutter pour la conscientisation.

\section{Rôle principal du travailleur social}

- Rôle de conscientisation auprès des usagers des services sociaux.

\section{Focus d'intervention}

- Responsabilisation du client dans sa démarche de résolution de problèmes ; 
- Création d'une alliance avec le client ;

- Obtention d'un effet multiplicateur ;

- Action au niveau des facteurs structurels.

\section{Composantes de l'action professionnelle}

- Détection, conscientisation et formation d'alliés dans la communauté ;

- Mise sur pied de ressources gérées par la communauté ;

- Habiletés à travailler en équipe ;

- Habiletés à travailler par projets à partir du regroupement des clientèles;

- Présuppose une délimitation territoriale.

\section{Principales constatations}

Le principal objectif de cette section est de répondre à la question suivante : est-ce que l'expérience réalisée au C.S.S.Q.-Charlevoix répond aux différents critères d'un mode d'approche intégré, tant au niveau du but fixé, du principal rôle du travailleur social, du focus d'intervention qu'au niveau des différentes composantes de l'action professionnelle?

Ainsi, considérant :

- que les problématiques auxquelles s'adresse l'approche intégrée dans Charlevoix (individus, groupes ou communautés aux prises avec des problèmes de pauvreté, de chômage, de délinquance, de monoparentalité, ayant des difficultés familiales ou conjugales) se retrouvent parmi celles les plus souvent identifiées par les tenants de l'approche structurelle (problèmes quotidiens d'individus, de familles ou de groupes aux prises avec la pauvreté, l'isolement, l'oppression, l'exclusion);

- que les aspects idéologiques liés à l'approche intégrée (conscientisation, solidarité avec les clients, lutte des classes, nouveau projet de société) s'inspirent grandement du postulat de base de l'approche structurelle (rapports entre les systèmes considérés comme antagonistes, opposés et inégalitaires);

- que le but poursuivi par les intervenants de Charlevoix (développement d'un pouvoir collectif et lutte pour la conscientisation) demande un changement radical, lequel est proclamé par les intervenants qui utilisent l'approche structurelle; 
- que le focus d'intervention de l'approche intégrée (responsabilisation du client, création d'une alliance, effet multiplicateur, action sur les facteurs structurels) s'associe de près à ceux des approches centrée sur la tâche et structurelle, tant au niveau des valeurs dominantes qu'au niveau de son orientation ;

l'expérience réalisée au C.S.S.Q.-Charlevoix répond aux différents critères d'un mode d'approche intégré et ce, aussi bien au niveau du but fixé, du rôle professionnel du travailleur social, qu'à celui du focus d'intervention. De plus, nous pouvons observer une influence directe de l'approche structurelle, qui semble avoir servi de toile de fond particulièrement au niveau de l'idéologie de base de l'approche intégrée.

Mais c'est au niveau des composantes de l'action professionnelle que se définit le cadre innovateur de cette expérience.

Revenons sur certains éléments qui définissent l'innovation sociale. On sait que le terme " innovation sociale " recouvre des pratiques qui ne sont pas forcément nouvelles; que toute action visant directement ou indirectement à l'adéquation des réponses données par un groupe d'individus aux besoins reconnus comme siens concerne l'innovation sociale ; qu'elle se définit soit dans le champ de la nouveauté (invention de solutions nouvelles), soit dans celui des solutions classiques, par leur adaptation à la transformation ou à la diversification des besoins. En fait, innover c'est avant tout agir.

Ainsi, les diverses composantes de l'action professionnelle de l'approche intégrée ne s'inscrivent pas dans un cadre totalement nouveau puisque, entre autres, le travail en équipe fait partie des composantes de l'action de l'approche centrée sur la tâche tandis que la notion de projet d'intervention a été empruntée à l'action communautaire. Toutefois, c'est dans l'utilisation de techniques et de modalités d'action, qui redonnent aux personnes concernées l'usage de leur capacité d'autonomie et dans lesquelles les médiations sont réduites au minimum afin de permettre aux personnes de se prendre en charge par elles-mêmes, que se définit le cadre innovateur de cette expérience d'intervention sociale.

\section{Les aspects positifs et les difficultés que soulève l'approche intégrée}

\section{Les aspects positifs}

Les intervenants comme les gestionnaires consultés s'entendent pour dire que l'expérience de l'approche intégrée au C.S.S.Q.-Charlevoix 
a su insuffler une motivation nouvelle aux praticiens. Le fait de vivre des expériences positives et valorisantes leur a redonné le goût de la pratique du service social et l'occasion de se réapproprier certains de ses éléments, tant sur le plan théorique que méthodologique. De plus, parce que cette approche laisse une marge de manœurre plus importante que les approches plus traditionnelles, la satisfaction et la valorisation que les intervenants ont pu en retenir étaient augmentées. Enfin, elle a permis de développer un esprit de travail positif favorisant ainsi une créativité plus grande aussi bien chez les praticiens que chez l'usager des services.

Sur le plan méthodologique, les intervenants prétendent que l'approche intégrée leur a permis de sortir d'un cadre d'intervention basé strictement sur l'intervention individuelle. De plus, le fait de se questionner régulièrement sur le pourquoi et le comment d'une intervention leur a permis d'élargir leur horizon professionnel au moyen d'un perfectionnement plus approprié et de devenir plus critique par rapport aux actions qui doivent être posées.

Les gestionnaires, quant à eux, parlent d'une décompartimentation de la pratique et d'un décloisonnement entre les services et les praticiens. Ainsi, le fait de référer à l'une ou l'autre des méthodes d'intervention semble avoir permis une familiarisation plus grande avec l'ensemble des méthodes et avec un plus grand nombre de techniques et de modèles.

Pour le praticien, cela signifie la capacité d'atteindre les buts et les objectifs fixés, en faisant usage des trois méthodes quand une seule n'est pas suffisante; une plus grande satisfaction dans l'atteinte de ces buts et objectifs; plus d'échanges avec les autres intervenants sur une même problématique ; plus de support apporté par le groupe (l'équipe) et la communauté; et finalement, une meilleure intégration dans la communauté.

Pour la clientèle, l'approche intégrée a su améliorer la qualité des services en offrant plus de continuité dans la dispensation des services eux-mêmes et a permis d'offrir, aux usagers des services sociaux, des ressources spécialisées capables de prendre en charge les bénéficiaires, d'une façon plus soutenue et plus globale. De plus, le support apporté par un groupe de bénéficiaires, l'établissement d'une relation d'aide plus souple, des appuis différents et plus nombreux favorisent normalement l'autonomie et la sociabilité.

Enfin, pour l'administration et l'organisme prestataire des services, le fait d'utiliser l'approche intégrée a pu, dans bien des cas, aider au règlement de diverses difficultés par et dans l'équipe, diminuant ainsi la lourdeur de la gestion. On parle alors d'une meilleure répartition du 
pouvoir entre l'administration et les intervenants facilitant, par conséquent, l'atteinte de la mission de l'organisme.

On ne relève pas d'économie de temps dans la réalisation des interventions, mais une capacité à satisfaire la clientèle du C.S.S.Q. en lui fournissant les outils nécessaires pour fonctionner plus longtemps et plus adéquatement. Par exemple, l'intervention reliée à un jeune en difficulté peut impliquer non seulement ce dernier et sa famille, mais aussi les ressources de l'école, de la municipalité et des organismes communautaires du milieu. Cette aide difficilement quantifiable est un gain pour l'organisme, pour les intervenants et les usagers des services sociaux.

\section{Les difficultés}

Les gestionnaires prétendent qu'il peut être difficile de transmettre l'idéologie de l'approche intégrée aux bénéficiaires des services, de partager avec eux cette nouvelle vision de la réalité sociale, parce qu'elle ne correspond pas vraiment à la conception qu'ils se font d'un service d'aide psychosociale. De plus, si l'application de l'approche est trop rigide, certains bénéficiaires pourraient recevoir des services sous une forme qui ne leur convient pas ou qu'ils ne désirent tout simplement pas, et se désintéresser graduellement des projets dans lesquels ils ont été amenés à participer. Ne pouvant s'assurer de leur collaboration, il pourrait devenir difficile pour les intervenants d'assurer une continuité dans la réalisation des divers projets.

L'intervention de groupe et collective amène des résistances de la part des gestionnaires parce qu'ils se demandent si les actions entreprises sont vraiment conformes au mandat d'aide psychosociale qui est confié aux centres de services sociaux. Par exemple, les interventions qui s'appuient davantage sur les ressources et les dynamismes des communautés dépassent les limites du travail de cas.

L'intervention de groupe et collective amène des résistances de la part de certains intervenants qui voyaient, eux aussi, s'éloigner les objectifs de base du C.S.S.Q. et qui se sentaient entraînés dans quelque chose qu'ils ne connaissaient pas. Par exemple, comment négocier avec une municipalité le développement d'une ressource de loisirs pour les jeunes âgés de douze à dix-huit ans.

Aussi bien intervenants que gestionnaires reconnaissent que l'une des principales difficultés réside dans le fait qu'il est plus difficile de quantifier, avec des unités de mesure appropriées, les interventions de groupe et communautaires que les interventions individuelles. Il devient, 
par conséquent, plus difficile pour les gestionnaires de justifier l'utilisation des budgets et d'exercer un certain contrôle sur les activités réalisées.

Le développement du travail d'équipe a nécessairement des implications au plan des méthodes de travail et des structures organisationnelles. Avec le temps, certains intervenants ont trouvé que le travail réalisé en équipe occasionnait de nombreuses pertes de temps, à cause de la fréquence trop grande des réunions. Il occasionnait aussi une perte d'autonomie et d'indépendance professionnelle. Selon eux, pour que l'expérience du travail en équipe soit valable, il faut que l'équipe soit supportante et compétente, que les buts visés par cette dernière soient clairs et qu'il y ait un encadrement professionnel adéquat.

Selon les intervenants et les gestionnaires, l'expérience de l'approche intégrée est exigeante sur le plan de l'engagement personnel aussi bien que professionnel. Tout d'abord, elle exige un investissement en temps et énergie considérable, parfois même allant empiéter sur le temps prévu pour la vie familiale ou les activités de loisirs. Puis elle exige plus de sécurité et d'autonomie professionnelle que les autres formes d'interventions, parce que l'action se situe toujours aux trois niveaux d'interventions, soit parallèlement ou en continuité selon la nature des difficultés. Elle peut donc être menaçante pour de jeunes professionnels insuffisamment autonomes pour se lancer dans ce type d'intervention.

Enfin les gestionnaires se demandent comment faire pour évaluer les résultats obtenus par l'approche intégrée, comment s'assurer qu'ils sont vraiment attribuables à ce mode d'intervention. De plus, il leur semble difficile de mesurer l'impact réel de l'approche sur la résolution des problèmes des usagers parce que l'intervention réalisée visait non seulement les clientèles des charges de cas (" caseloads"), mais aussi des gens qui n'en provenaient pas.

\section{Conclusion}

Peut-on généraliser l'approche intégrée ? Dans quel milieu institutionnel, dans quel milieu géographique peut-elle évoluer? Quelles sont les conditions reliées à sa généralisation? Ce sont là les dernières questions auxquelles nous allons tenter de répondre.

Ainsi, il semble, d'après les intervenants et les gestionnaires, que l'approche intégrée peut être généralisée aussi bien à l'intérieur des cadres d'un centre de services sociaux que d'un centre local de services communautaires. Ce sont les modalités d'application qui vont permettre ou non sa réalisation plutôt que la mission spécifique des établissements.

Par exemple, il faut s'assurer que :

- l'approche intégrée ne soit pas utilisée pour fuir l'intervention individuelle, les trois méthodes étant aussi importantes les unes que les autres ; 
- la philosophie sous-jacente soit clairement exprimée ;

- les projets élaborés répondent aux besoins réels des usagers;

- les projets de groupe ne deviennent pas contraignants pour les intervenants;

- les professionnels s'assurent d'une prise en charge des projets par la communauté ;

- le temps accordé aux discussions de cas, à l'élaboration et l'évaluation des projets ne finisse pas par réduire le temps d'exécution de l'intervention proprement dite ;

- cette expérience soit supportée par un encadrement professionnel fort ;

- il y ait une acceptation du principe par les gestionnaires ;

- ce soit une volonté de l'établissement ;

- l'on puisse dégager des indices de mesure pour une meilleure utilisation ;

- la structure interne du groupe (comités, règlements) ne vienne pas étouffer le fonctionnement du groupe;

- les exigences du travail (réunions le soir et les fins de semaine) tiennent compte des réalités familiales et personnelles;

- les praticiens connaissent les fondements de cette approche (idéologie, cadre d'analyse, processus d'intervention);

- les praticiens aient une bonne connaissance de leur territoire d'intervention;

- les praticiens reconnaissent que les clients peuvent choisir le mode d'intervention qui leur convient le mieux;

- les praticiens prennent garde à toute forme d'épuisement professionnel;

- l'équipe de travail demeure un lieu de ressourcement professionnel;

- l'expérience de travailler en équipe ne soit pas menaçante pour le praticien;

- l'équipe soit relativement stable;

- il y ait partage d'un même intérêt pour ce type d'expérience au sein de l'équipe;

- chacun des membres de l'équipe conserve son autonomie professionnelle. ${ }^{7}$

Il semble aussi que le milieu géographique (région urbaine versus région rurale) ne soit pas une limite à l'utilisation et à la généralisation de l'approche intégrée. Bien plus que le milieu lui-même, l'interaction entre l'intervenant et la population cible de son territoire est un facteur très significatif. 


\section{APPENDICE A}

\section{Approches intégrées selon les principales dimensions}

\begin{tabular}{|c|c|c|}
\hline $\begin{array}{l}\begin{array}{c}\text { Approche } \\
\text { généraliste }\end{array} \\
\text { Dimension } \\
\text { à l'étude }\end{array}$ & $\begin{array}{l}\text { Approche centrée } \\
\text { sur la tâche }\end{array}$ & $\begin{array}{l}\text { Approche } \\
\text { systémique }\end{array}$ \\
\hline But du service social & $\begin{array}{l}\text { - Définir les tâches du } \\
\text { client, du profession- } \\
\text { nel et assembler les } \\
\text { connaissances et les } \\
\text { habiletés requises pour } \\
\text { le parachèvement de } \\
\text { ces tâches. }\end{array}$ & $\begin{array}{l}\text { - Intervenir sur et en- } \\
\text { tre les systèmes de } \\
\text { façon à créer un im- } \\
\text { pact des "change-agent } \\
\text { systems " sur les autres } \\
\text { systèmes. }\end{array}$ \\
\hline $\begin{array}{l}\text { Rôle principal du tra- } \\
\text { vailleur social }\end{array}$ & $\begin{array}{l}\text { - Facilitateur qui aide } \\
\text { le client dans la réa- } \\
\text { lisation de sa tâche. }\end{array}$ & $\begin{array}{l}\text { - Médiateur entre les } \\
\text { systèmes. }\end{array}$ \\
\hline Focus d'intervention & $\begin{array}{l}\text { - Les valeurs qui sont } \\
\text { une base commune } \\
\text { sur laquelle reposent } \\
\text { les buts à atteindre et } \\
\text { les tâches à accomplir. } \\
\text { - Autodétermination } \\
\text { du client. } \\
\text { - Croissance du client. } \\
\text { - Capacité de s'en sortir. }\end{array}$ & $\begin{array}{l}\text { - Maximisation du de- } \\
\text { gré d'atteinte des buts } \\
\text { de chaque système } \\
\text { (système fonctionnel). }\end{array}$ \\
\hline
\end{tabular}




\begin{tabular}{|c|c|c|}
\hline $\begin{array}{c}\text { Approche } \\
\text { psychosociale }\end{array}$ & $\begin{array}{c}\text { Approche } \\
\text { structurelle }\end{array}$ & $\begin{array}{l}\text { Approche intégrée } \\
\text { de Charlevoix }\end{array}$ \\
\hline $\begin{array}{l}\text { - Intervenir sur les } \\
\text { rôles, les structures } \\
\text { des petits groupes et } \\
\text { les processus d'in- } \\
\text { fluence sociale. }\end{array}$ & $\begin{array}{l}\text { - Développer des rap- } \\
\text { ports plus égalitaires } \\
\text { entre les systèmes. } \\
\text { - Changement radical } \\
\text { à moyen et long } \\
\text { terme. }\end{array}$ & $\begin{array}{l}\text { - Développer un pou- } \\
\text { voir collectif et lutter } \\
\text { pour la conscientisa- } \\
\text { tion. }\end{array}$ \\
\hline $\begin{array}{l}\text { - Expert qui utilise } \\
\text { diverses informations } \\
\text { pour produire des } \\
\text { changements avec } \\
\text { ou sur le client. }\end{array}$ & $\begin{array}{l}\text { - Conseiller, courtier, } \\
\text { médiateur, avocat, } \\
\text { personne-ressource, } \\
\text { expert. } \\
\text { - C'est un allié du } \\
\text { client avec qui il éta- } \\
\text { blit un rapport égali- } \\
\text { taire de type dialo- } \\
\text { gique. }\end{array}$ & $\begin{array}{l}\text { - Conscientisation } \\
\text { auprès des usagers } \\
\text { des services sociaux. }\end{array}$ \\
\hline $\begin{array}{l}\text { - Fonctionnement } \\
\text { social et fonctionne- } \\
\text { ment individuel. }\end{array}$ & $\begin{array}{l}\text { - Les relations entre les } \\
\text { individus, groupes, } \\
\text { collectivités avec eux- } \\
\text { mêmes et avec leur } \\
\text { situation socio-poli- } \\
\text { tique et économique. }\end{array}$ & $\begin{array}{l}\text { - Responsabilisation } \\
\text { du client dans sa } \\
\text { démarche de résolu- } \\
\text { tion de problèmes. } \\
\text { - Création d'une allian- } \\
\text { ce avec le client. } \\
\text { - Action au niveau des } \\
\text { facteurs structurels. }\end{array}$ \\
\hline
\end{tabular}




\section{APPENDICE A (suite)}

\section{Approches intégrées selon les principales dimensions}

\begin{tabular}{|l|c|c|}
\hline $\begin{array}{c}\text { Approche } \\
\text { Diménsion } \\
\text { à l'étude }\end{array}$ & $\begin{array}{c}\text { Approche centrée } \\
\text { sur la tâche }\end{array}$ & $\begin{array}{c}\text { Approche } \\
\text { systémique }\end{array}$ \\
\hline $\begin{array}{l}\text { Composantes d'une } \\
\text { action professionnelle }\end{array}$ & $\begin{array}{c}\text { - Équipe profession- } \\
\text { nelle; } \\
\text { - Un ustaff " qui plani- } \\
\text { fie et dirige. } \\
\text { - Délégation de cer- } \\
\text { tains rôles de gestion } \\
\text { à l'équipe. }\end{array}$ & $\begin{array}{c}\text { - Actions au niveau de } \\
\text { la communication, du } \\
\text { feedback, de la négo- } \\
\text { ciation. } \\
\text { - Habiletés au niveau } \\
\text { du "problem-solving" } \\
\text { en relation avec les } \\
\text { problèmes de fonc- } \\
\text { tionnement systémi- } \\
\text { que et d'échange in- } \\
\text { ter-système. }\end{array}$ \\
& & \\
& \\
&
\end{tabular}

SOURCES: La conception de ce tableau, de même que le contenu des trois premières approches sont tirés de : F. OUELLET, "Une conception de la formation et de l'évaluation en service social ", dans: Regroupement des unités de formation universitaire en travail social du Québec, La formation en travail social au Québec: d'une École à l'autre, Montréal, 1984, pp. 69-90. Le contenu sur l'approche structurelle est tiré d'un document miméographié de M. LAFOREST, "Grille pour situer et préciser les pratiques dites intégrées", Québec, École de service social de l'Université Laval, 1985. Le contenu sur l'approche intégrée de Charlevoix provient de discussions avec les intervenants du C.S.S.Q., territoire de Charlevoix, avril 1986. 


\begin{tabular}{|c|c|c|}
\hline $\begin{array}{c}\text { Approche } \\
\text { psychosociale }\end{array}$ & $\begin{array}{l}\text { Approche } \\
\text { structurelle }\end{array}$ & $\begin{array}{l}\text { Approche intégrée } \\
\text { de Charlevoix }\end{array}$ \\
\hline $\begin{array}{l}\text { - Renforcement } \\
\text { - Désensibilisation } \\
\text { - Redéfinition de situa- } \\
\text { tion } \\
\text { - Recentralisation de } \\
\text { l'attention } \\
\text { - Restructuration de } \\
\text { groupe } \\
\text { - Réappariement de l'in- } \\
\text { dividu et du groupe. }\end{array}$ & $\begin{array}{l}\text { - Actions au niveau de } \\
\text { la conscientisation, } \\
\text { du regroupement et } \\
\text { de l'action sociale. } \\
\text { - Habiletés pour sou- } \\
\text { lager les tensions } \\
\text { immédiates, orientér } \\
\text { vers conscientisation, } \\
\text { solidarisation et mobi- } \\
\text { liser vers le dévelop- } \\
\text { pement de groupes } \\
\text { de pression (counse- } \\
\text { ling, courtage, média- } \\
\text { tion, négociation, ad- } \\
\text { vocacy, etc.). Ce qui } \\
\text { implique des actions } \\
\text { auprès des individus, } \\
\text { familles, groupes qui } \\
\text { vivent les problèmes } \\
\text { de même que les ac- } \\
\text { tions auprès des orga- } \\
\text { nisations, des res- } \\
\text { sources communau- } \\
\text { taires et des instances } \\
\text { sociétales concer- } \\
\text { nées par les dits pro- } \\
\text { blèmes. }\end{array}$ & $\begin{array}{l}\text { - Détection, conscien- } \\
\text { tisation et formation } \\
\text { d'alliés dans la com- } \\
\text { munauté. } \\
\text { - Mise sur pied de res- } \\
\text { sources gérées par la } \\
\text { communauté. } \\
\text { - Habiletés à travailler } \\
\text { en équipe, par pro- } \\
\text { jets, à partir du re- } \\
\text { groupement des clien- } \\
\text { tèles. } \\
\text { - Délimitation territo- } \\
\text { riale. }\end{array}$ \\
\hline
\end{tabular}




\section{Notes et références}

1 Auclair, René et Lampron, Christiane. Approche intégrée: une innovation dans la dispensation des services sociaux. Recherche subventionnée par le Conseil québécois de la recherche sociale, Québec, 1987, pages 27-63.

2 Dans ce rapport de recherche, le générique masculin est utilisé sans aucune discrimination et uniquement dans le but d'alléger le texte.

3 Dorénavant, le Centre de services sociaux de Québec, territoire de Charlevoix, sera identifié par le sigle suivant: C.S.S.Q.-Charlevoix.

${ }^{4}$ Tremblay, Pierre. L'approche intégrée des trois méthodes de service social dans Charlevoix. Texte miméo, École de service social, Université Laval, Québec, avril 1982, p. 8.

5 Tiré de : Jules Perron. "La distribution des services sociaux", dans Modèles et analyses du travail social au Québec. Centre de recherche sur le Travail Social, Université de Caen, 1982, p. 53. (Avec l'autorisation de l'auteur et du Centre de Recherche sur le Travail Social.)

6 Rose, H. Méthodologie et stratégie de l'organisation du travail. Paris, Dunod, 1964.

7 Les conditions de généralisation ont été formulées à partir des propos tenus par les intervenants et les gestionnaires rencontrés. 


\section{APPENDICE B}

\section{Guide d'entrevue}

\section{Praticiens}

\section{Historique}

1. Quels sont les facteurs qui expliquent le développement de l'approche intégrée dans Charlevoix?

a) le contexte socio-économique de Charlevoix?

b) le contexte organisationnel?

c) le désir d'expérimenter un mode d'approche innovateur?

d) l'arrivée d'un nouveau chef d'équipe?

e) autres?

\section{Cadre général d'intervention}

2. Quelle est la philosophie de l'approche intégrée?

- principes ou hypothèses qui sous-tendent l'action

- niveaux d'action

Réformes administratives nécessitées par cette approche à Charlevoix

3. Quels ont été les changements administratifs nécessaires à la mise sur pied de l'approche?

- mise sur pied de nouvelles structures de fonctionnement?

— redéfinition des rôles administratifs?

4. Cette approche vous a-t-elle demandé de modifier

- certaines attitudes?

- l'organisation de votre travail ?

— certaines tâches?

- autres?

Modes d'intervention

5. Quelles activités avez-vous accomplies auprès de la clientèle?

a) interventions cliniques auprès de personnes (charge habituelle de cas) 
b) projets : avez-vous combiné différents modes d'intervention (individuel et groupe ; individuel et intervention communautaire, etc.)

c) autres types d'intervention

6. Avez-vous travaillé seul ou avec d'autres personnes dans chacun des projets?

Objectifs poursuivis

7. Quels étaient les objectifs visés dans chacun des projets auxquels vous avez été associé?

— objectifs à long terme? court terme?

Résultats obtenus

8. Quels ont été les objectifs réalisés dans chacun des projets?

9. Quels ont été les objectifs non réalisés dans chacun des projets et pourquoi?

10. Quels ont été les objectifs réalisés qui n'avaient pas été prévus?

11. Combien de personnes ont été rejointes par le projet?

- directement

- indirectement

12. Quelle a été la durée du projet?

\section{Possibilités de généralisation}

13. Quelles sont les possibilités de généralisation de cette approche?

- est-elle applicable dans n'importe quel milieu géographique?

- est-elle applicable dans n'importe quel milieu institutionnel ?

- est-elle applicable à n'importe quelle problématique?

14. Quelle sont les conditions nécessaires au fonctionnement de cette approche?

15. Si c'était à refaire, quels sont les façons de procéder qui, selon vous, devraient être modifiées? 


\section{APPENDICE C}

\section{Guide d'entrevue}

\section{Gestionnaires}

\section{Objectifs de l'approche intégrée}

1. Quels étaient, selon vous, les objectifs poursuivis dans le cadre de l'approche intégrée?

\section{Rôle joué par l'administrateur}

2. Quel rôle avez-vous joué par rapport au développement de cette approche?

- facilitateur

- médiateur

- etc.

\section{Opinions sur l’approche intégrée}

3. Quels étaient, selon vous, les avantages de cette approche

- pour la clientèle?

- pour les intervenants?

- pour la structure administrative?

- autres

4. Quels étaient, selon vous, les inconvénients de cette approche - pour la clientèle?

- pour les intervenants?

- pour la structure administrative?

- autres

\section{Possibilités de généralisation}

5. Cette approche est-elle applicable

- dans un Centre de services sociaux?

- dans une autre institution qui distribue des services sociaux ?

6. Quels sont les conditions nécessaires au fonctionnement de cette approche? 